\title{
A second shot at sight using a fully organic retinal prosthesis
}

\begin{abstract}
A new photovoltaic device using organic polymers and a silk substrate can bring back vision to blind rats and may provide a much simpler retinal prosthetic for future use in humans.
\end{abstract}

Larry Hester began to notice trouble in his mid-thirties; his vision was giving way and there was nothing he, nor any doctors, could do about it. His condition, retinitis pigmentosa, robs about 1 in 4,000 people of their sight through gradual degeneration of retinal cells, and reflects an uncomfortable fact about vision: its biological foundation is a house of cards. Photoreceptors, the kickoff point for vision in the retina, are extremely finicky: disruption to any one of 150-200 different genes affecting their function can cause them to throw in the towel.

Since the early 1990's, scientists have been developing retinal prostheses, sometimes known as 'bionic eyes', to help give people like Larry back their vision, or at least some part of it. These devices, which bypass lost or damaged photoreceptors using electrode arrays implanted directly in the retina, can generate basic visual sensations called phosphenes. These phosphenes can range from primitive (lights on vs. lights off) to relatively complex (letters and shapes), depending on several factors, including the degree of damage to the diseased retina.

Although retinal prostheses offer important relief for people with no other treatment options, current devices are not without their shortcomings. As one might imagine, they're very complex: in addition to the intraocularly placed electrode array, there are a slew of extraocular components that must be ligated to the sclera - the white outer layer of the eyeball-or attached to accompanying glasses that patients must wear for the prosthetic to detect light and work properly; and with each additional component comes additional risks for things to go wrong.

Fabio Benfenati, Professor at the University of Genoa, Italian Institute of Technology, Italy, thinks we can do better, and a recent article published by his group in Nature Materials shows how (Nat. Mater. doi:10.1038/ nmat4874; published online 06 March 2017).
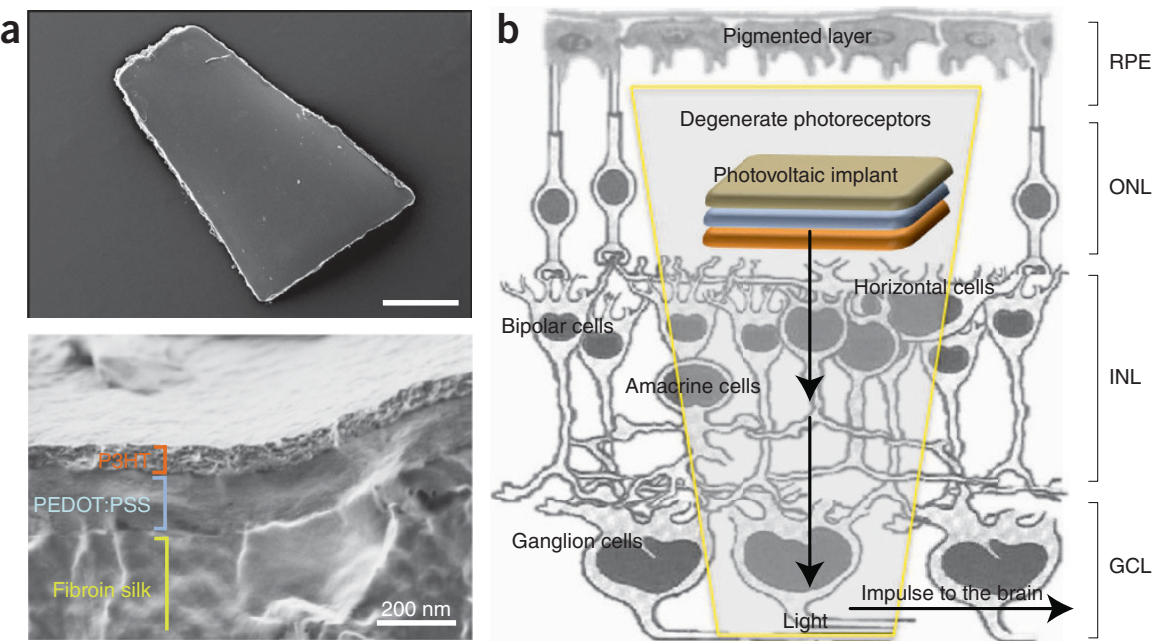

The device and subretinal implant. (a) Scanning electron microscopy image of the full prosthetic implant, and (b) schematic of the implant strategy in the retina. Adapted from Nat. Mater. doi:10.1038/nmat4874.

"We've been developing new lines of neuronal interfaces, using a variety of what we call 'smart materials,' says Benfenati. These materials include organic polymers that were developed for solar cells in the lab of Guglielmo Lanzani, a physicist and collaborator at the Center for Nano Science and Technology in Milan, Italy. Using this technology, Benfenati's group, which focuses on neurophysiology, joined forces with Lanzani and experts in biotechnology and an ophthalmology team led by Grazia Pertile to create a fully organic photovoltaic retinal prosthesis that can restore basic vision in rat models of degenerative blindness.

Unlike current retinal prostheses, with separate elements for each step of light detection, transmission and electrical stimulation of the retina, the photovoltaic device described in their new paper is completely self-contained, eliminating the need for any additional components. Fabricated from thin layers of photoactive polymers ( $\sim 800 \mathrm{~nm}$ thick) attached to a soft and highly flexible silk substrate, the device is a good match for long-term companionship with the delicate retina.

In their paper, the team shows that their novel retinal prosthetic is capable of restoring basic visual reflexes, like pupillary constriction, in Royal College of Surgeons rats, a well-established model of retinitis pigmentosa. Additionally, the device can generate electrical responses to light in the visual cortex of blind rats, and even restore the animal's natural behavioral aversion to light when given a choice between light and dark boxes.

Benfenati is hopeful his group will be able to move their new prosthethics into humans soon for clinical trials, but in the meantime they are continuing in vivo testing using pig models, which have eyes more similar to humans compared to rats. "The pig eye has the same dimensions as the human eye, and very similar structure, in terms of presence of cones and high resolution vision", says Benfenati. He also says that pig eyes are extremely sensitive to surgical insult, making it a good testing ground to determine the safety of their implant. "It's a difficult and tricky model, but if we get good results in such risky and challenging conditions, then probably the human eye would be relatively safe."

In addition to helping patients, human trials would also provide new data to continue improving the device. "Once proving safety in pigs and moving into human trials, we could get a lot more information that the animals cannot give, so that will be an important turning point."

Dustin M. Graham 